\title{
Rapid postoperative onset of neurological dysfunction
}

\author{
J E Grey, P G Flanagan
}

A 69-year-old woman with severe rheumatoid arthritis was admitted with rigors 3 weeks following a left total knee replacement. She had a severe deforming arthropathy with an unstable cervical spine and had had multiple joint replacements. She was being treated with prednisolone and penicillamine. The left knee was slightly warm but there was no effusion or restriction of movement. There were no signs of meningitis. She had a superficial pressure sore over the 8 th thoracic vertebra. Urinalysis showed proteinuria and she had a leucocytosis. She was treated with co-amoxiclav ( $625 \mathrm{mg} 8$ hourly, oral) for suspected urinary tract infection and flucloxacillin (500 mg 6 hourly, oral) for possible staphylococcal septicaemia in view of the pressure sore and recent joint replacement. Urine and blood cultures were subsequently negative. Her condition deteriorated over the next day and her antibiotic regimen was changed to cefotaxime ( $1 \mathrm{~g} 8$ hourly, intravenously) and vancomycin ( $1 \mathrm{~g} 12$ hourly, intravenously) as empirical treatment of septicaemia of undetermined origin. She responded well, but four days later she dislocated her right total hip replacement whilst turning over in bed (an event which had happened twice previously). The hip was relocated under spinal anaesthetic using marcaine in dextrose. Initially the anaesthetist aspirated clear cerebrospinal fluid (CSF) but aspiration following introduction of marcaine revealed purulent CSF. Examination of the CSF revealed 40 red blood cells $/ \mathrm{mm}^{3}, 2920$ white blood cells/ $\mathrm{mm}^{3}$ (98\% neutrophils) and Gram-positive cocci were seen on microscopy.

A few hours postoperatively she became unrousable and had developed neck stiffness.

University Hospital of Wales, Heath Park, Cardiff CF4 4XW, South Glamorgan, Wales, UK

Department of Integrated Medicine J E Grey

Department of Clinical Microbiology

P G Flanagan

Accepted 16 December 1997 Neurological examination at this time was very difficult in view of her condition, though her left plantar reflex was equivocal. However, because of the paucity of neurological signs, the neuroradiologists felt that magnetic resonance imaging (MRI) was not indicated. Within a week, her ankle, knee and plantar responses became absent. Sensory examination remained difficult to interpret because of poor cooperation but it was felt that there was abnormal sensation from L4 down.

\section{Questions}

1 What is the diagnosis?

2 What other conditions are in the differential diagnosis?

3 What is the treatment of choice for this patient? 


\section{Answers}

QUESTION 1

Spinal epidural abscess (SEA). CSF taken intraoperatively yielded a pure growth of methicillinresistant Staphylococcus aureus (MRSA). A initial diagnosis of MRSA meningitis was made immediately post-operation and antibiotic therapy was changed to vancomycin (1 g 12 hourly, intravenously) and rifampicin (600 $\mathrm{mg} 12$ hourly, oral). However a neurologist who examined the patient $48 \mathrm{~h}$ later felt that she had a loculated cauda equine SEA. Five days later an MRI scan was performed and provided an equivocal result ('cauda equina roots are illdefined and suspicious'). We postulate that a SEA developed secondary to epidural anaesthesia, caused by MRSA that was colonising the skin at the puncture site. However, the possibility that she acquired the infection via blood from other sites cannot be excluded. When she underwent her spinal puncture for hip re-location (4 weeks after the original operation), we postulate that the needle penetrated the loculated SEA, causing pus to enter the subarachnoid space, precipitating the sudden worsening of her condition. SEAs usually extend for four or five vertebral segments, but may extend for the whole length of the spinal cord. ${ }^{1}$ MRSA colonisation was documented in several different body sites within a week of her initial CSF isolate, including a pressure sore over the 8th thoracic vertebra. The patient responded well to the treatment and recovered fully after 3 weeks of therapy

QUESTION 2

Primary postoperative meningitis (without pre-existing suppuration). This diagnosis was less likely due to the relatively short interval between the operation and the onset of clinical symptoms. $^{2}$

\section{QUESTION 3}

Urgent neurosurgical referral and antibiotics with anti-staphylococcal activity. The CSF Gram stain suggested staphylococcal sepsis, which was shown to be caused by MRSA. Vancomycin is the drug of choice for MRSA infections. ${ }^{3}$ However, sustained improvement was achieved only after her second spinal puncture and the administration of intravenous vancomycin and oral rifampicin. This combination has been used to treat serious MRSA infections such as endocarditis, ${ }^{3}$ and some in-vitro studies have shown synergism between these agents against MRSA. ${ }^{4}$ There are few data on the clinical efficacy of this combination in comparison to vancomycin alone, or other agents, in the treatment of MRSA infections of the central nervous system, ${ }^{56}$ due to the rarity of the condition. However, there are reports demonstrating the enhanced clinical efficacy of vancomycin plus rifampicin to treat methicillin-resistant Staphylococcus epidermidis

1 Redekop G, Del Maestro R. Diagnosis and management of spinal epidural abscess. Can 7 Neurol Sci 1992;19: $181-7$ meningitis, particularly when the vancomycin is given by the intraventricular route. ${ }^{7}$ Given the poor penetration of vancomycin into CSF and the excellent penetration of rifampicin, the addition of the latter agent to the antimicrobial regimen, would seem rational.

With the inexorable increase in the incidence of MRSA infection in the UK, vancomycin, with or without rifampicin, may soon become the antibiotic of choice in cases of suspected staphylococcal infection in hospitals with high endemic rates of MRSA infection/colonisation This has obvious cost implications for clinical practice.

\section{Discussion}

SEA is a comparatively rare condition in which $S$ aureus is the most common causative organism. ${ }^{1}$ Spinal epidural infection usually occurs as a result of either haematogenous spread from cutaneous infection, pyelonephritis, or pneumonia, or by direct extension from a local site, such as vertebral osteomyelitis. ${ }^{1}$ It has also followed epidural anaesthesia, lumbar puncture and back trauma. ${ }^{8-10}$ Abscesses most commonly occur in the thoracic and lumbar regions, which have large epidural spaces with an extensive blood supply. ${ }^{1}$ Fever, spinal tenderness, and back pain are the most commonly reported clinical features of SEA. Our patient complained of spinal tenderness on palpation but no back pain and was apyrexial throughout. The absence of pain and temperature is consistent with previous reports of SEA. ${ }^{10}$ Prior to the advent of MRI and computed tomography, clinical investigation of suspected SEA included lumbar puncture and myelography, both of which can precipitate extension of the abscess into the subarachnoid space, causing septic meningitis. ${ }^{9}$

The treatment of choice for SEA remains urgent surgical drainage, combined with antibiotic therapy appropriate for the organisms isolated. A few case reports of SEA managed non-surgically have been reported. ${ }^{11} 12$

Colonisation and infection with MRSA is now a global problem in hospitalised patients and those in long-term residential care. ${ }^{213}$ However, MRSA meningitis, particularly in the absence of neurological prosthetic devices, is as yet uncommon, with only nine cases reported in the world literature between $1976-96 .{ }^{14} \mathrm{We}$ believe this is the first published case of an MRSA SEA.

\section{Final diagnosis}

Spinal epidural abscess caused by methicillinresistant Staphylococcus aureus.

We would like to thank Dr HGM Shetty for his helpful comments on the manuscript.

Keywords: spinal epidural abscess; MRSA

2 Kim J, van der Horst C, Mulrow C, Corey G. Staphylococcus aureus meningitis: review of 28 cases. Rev Infect Dis $1989 ; 11$ : 698-706. 
3 Duckworth G. Diagnosis and management of methicillin resistant Staphylococcus aureus infection. BMf 1993;307: 1049-52.

4 Keane CT, Cafferkey MT. Methicillin-resistant Staphylococcus aureus: a guide to epidemiology and control. Rev Med Microbiol 1991;2:50-6.

5 Vichyanond P, Olson LC. Staphylococcal CNS infections treated with vancomycin and rifampin. Arch Neurol treated with 6 .

6 Gordon JJ, Harter DH, Phair JP. Meningitis due to Staphylococcus aureus. Am $\mathcal{F}$ Med 1985; 78:965-70.

7 Ferree B, Stambough JL, Greiner AL. Spinal epidural abscess. A case report and literature review. Orthopaedic Rev abscess. A case

8 Hoefnagels WA, van Poucke GE, Casselman JW, van Kleef JG, Vannerem LT. An abscess as an unexpected complication following epidural anaesthesia. Ned Tijdschr Geneeskd 1994;138:580-2
9 Baker A, Ojemann RG, Swartz M, Richardson EP. Spinal epidural abscess. N Engl f Med 1975;293:463-8.

10 Dei-Anang K, Hase U, Schurmann K. Epidural spinal abscesses. Neurosurg Rev 1990;13:285-8.

11 Leys D, Lesoin F, Vidaud C, et al. Decreased morbidity from acute bacterial spinal epidural abscess using computed tomography and non-surgical management in selected patients. Ann Neurol 1985;17:350-5.

12 Mampalam TJ, Rosegay H, Andrew BT, et al. Non operative treatment of spinal epidural infections. $\mathcal{\Im}$ Neurosurg $1989 ; 71$ : 208-10.

13 Working party of British Society of Antimicrobial Chemotherapy and Hospital Infection Society. Guidelines on the therapy and Hospital Infection Society. Guidelines on the control of methicillin resistant Staphylococt

14 Sakamoto T, Kikuchi K, Mineura K, Kowada M, Nakagomi $O$. MRSA meningitis in postoperative patients. Report of 4 cases. $\mathfrak{f p}_{p} \mathcal{\Im}$ Antibiot 1990;43:1137-42.

\title{
Hypertension and renal failure
}

\author{
D J A Goldsmith, G Hamilton
}

A 74-year-old man with a history of intermittent claudication, cigarette-smoking, hypertension, and a successful recent right nephro-ureterectomy for a right ureter transitional cell carcinoma, presented with abnormal renal function and hypertension (plasma creatinine $228 \mu \mathrm{mol} / 1$ and blood pressure $200 / 100 \mathrm{mmHg}$ on quinapril $20 \mathrm{mg}$ ). Fundoscopy showed grade II hypertensive changes. There was no abdominal bruit. There was no blood and only a trace of protein on urine reagent strip testing; phase-contrast urinary sediment microscopy was normal; 24-h protein excretion was $0.2 \mathrm{~g}$ (normal $<0.2 \mathrm{~g} / 24 \mathrm{~h}$ ), and creatinine clearance was $36 \mathrm{ml} / \mathrm{min}$. Prior to the right nephrectomy, renal function had been abnormal (plasma creatinine $184 \mu \mathrm{mol} / \mathrm{l}$ ).

Ultrasound of his abdomen showed a nonobstructed $9.3 \mathrm{~cm}$ left kidney with increased echogenicity and reduced cortical thickness. Isotope renography while on an angiotensin-converting

Trafford Department of Renal Medicine, Royal Sussex County Hospital, Brighton, East Sussex BN2 5BE, UK

D J A Goldsmith

University

Department of

Surgery, Royal Free

Hospital, Pond St,

London NW3 2QG, UK

G Hamilton

Accepted 19 November 1997 enzyme (ACE) inhibitor showed no activity on the right (nephrectomy) and definite but sluggish excretion by the left kidney. Frusemide $40 \mathrm{mg} /$ day was added, which reduced blood pressure a little $(175 / 90 \mathrm{mmHg})$, but caused plasma creatinine to rise from 241 to $312 \mu \mathrm{mol} / 1$.

Quinapril was continued at $20 \mathrm{mg}$ daily and lacidipine $4 \mathrm{mg}$ was added. The patient could not tolerate any strength of any other calcium channel blocker, nor a beta- or an alpha-blocker. Blood pressure rose further. Quinapril was increased to $40 \mathrm{mg} /$ day, and plasma creatinine rose again to $386 \mu \mathrm{mol} / 1$. Echocardiography showed significant left ventricular hypertrophy and diastolic dysfunction.

\section{Questions}

1 Is it likely that this patient has glomerulonephritis?

2 What is the most probable reason for the raised blood pressure and worsening renal function?

3 What options are available for effecting an improvement? 